ACTA UNIVERSITATIS LODZIENSIS

Folia Litteraria Romanica 15, 2020

https://doi.org/10.18778/1505-9065.15.17

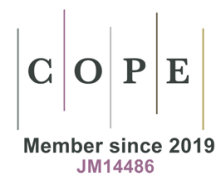

Denis Reynaud

Université Lumière Lyon 2

denis.reynaud@univ-lyon2

\title{
Jacques Sternberg : le mépris par alphabet
}

\begin{abstract}
RÉSUMÉ
Jacques Sternberg (1923-2006) fut une figure singulière de la scène littéraire parisienne dans les années 1960-1970, aujourd'hui surtout connue pour sa collaboration avec Alain Resnais et Roland Topor. En 1973, il publie un Dictionnaire du mépris où, en 443 entrées (d'Absurde à Zoo en passant par Minable), il exprime sa détestation de l'humanité en général et de la France pompidolienne en particulier. Le livre, qui sera suivi en 1985 par un Dictionnaire des idées revues, est rarement drôle. Son principal intérêt réside dans le recours à la forme alphabétique (empruntée à Voltaire, Flaubert et Ambrose Bierce) et dans son titre. Sternberg, qui prétend ne pas s'intéresser aux gens et ne pas aimer la littérature, se sent comme un prêtre qui ne croirait pas en Dieu. Mais pour lui le mépris est une passion revendiquée, étape nécessaire pour parvenir au « véritable humour », qui a peut-être ses racines dans l'expérience de l'horreur de la guerre et des camps où son père perdit la vie.
\end{abstract}

MOTS-CLÉS - Jacques Sternberg, Alphabet, Belgique, dictionnaire, humour, jeu de mots, juif, mépris, nazisme

"Jacques Sternberg: the ABCs of Contempt"

\section{SUMMARY}

Jacques Sternberg (1923-2006) was a singular figure of the Parisian literary life of the 60s and 70s, but today his name is mostly associated with Alain Resnais and Roland Topor. In 1973, he published a Dictionnaire du mépris, in which the humorist expressed his detestation of mankind in general and of French society under president Pompidou in particular. It was expanded in 1985 under the title Dictionnaire des idées revues. Neither book aims to be funny. Their main interest lies in their titles and in their form. Following in the footsteps of Voltaire, Gustave Flaubert and Ambrose Bierce, Sternberg establishes a strong connection between satire and the dictionary form; but his all-encompassing contempt - an essential ingredient of « true humour » - is of a different brand. He pretends to dislike not only people but literature. The key to this radicality is perhaps to be found in the young man's experience of the horrors of WW2 and of the camps where his father lost his life

KEYWORDS - Jacques Sternberg, Alphabet, Belgium, contempt, dictionary, humour, jew, nazism, puns 
Un homme vêtu d'un ciré de pêcheur breton, dont l'allure évoque un peu celle de Michel Houellebecq, pousse son Vélosolex en direction du café de Flore, où il a rendez-vous avec Roland Topor ${ }^{1}$. Ce piéton, c'est Jacques Sternberg (Anvers 1923 - Paris 2006), figure marginale de la scène littéraire parisienne, aujourd'hui quelque peu oubliée, qui, en deux réponses au journaliste qui l'accompagne, campe son personnage : « Les gens ne m'intéressent pas », puis : «J'aime pas la littérature ».

Ces répliques pourraient figurer dans le Dictionnaire du mépris ou dans le Dictionnaire des idées revues ${ }^{2}$, que Sternberg n'avait alors pas encore publiés, où on lit par exemple :

CAME. Écrire a été ma came pendant quarante ans, mais je n'ai jamais aimé les milieux littéraires ni les écrivains ni même la littérature en général. Et cela doit se sentir à travers ce que j'écris. Que penserait-on d'un prêtre qui ne monterait en chaire que pour nier l'existence de Dieu?

Au début des années 1960, Sternberg n'est guère connu que d'un petit cercle d'amateurs de ses nouvelles fantastiques et de science-fiction. Mais il vient de rencontrer Alain Resnais, avec qui il commence à travailler à la rédaction d'un scénario. Ce sera Je t'aime, je t'aime, sorti en 1968. Claude Ridder, le personnage principal du film, interprété par Claude Rich, est inspiré par la vie de Jacques Sternberg : comme lui, il a longtemps vécu d'emplois subalternes, dont celui d'emballeur et de sténodactylographe ; comme lui, c'est un survivant.

À la $10^{\mathrm{e}}$ minute du film, un savant déclare : "J'ai toujours pensé que nous devrions annexer à nos services techniques une équipe de romanciers, payés pour jeter des idées délirantes sur le papier ». C'est précisément la forme qu'a prise la collaboration de Sternberg et de Resnais. Pendant plusieurs années, le premier produisit de courtes scènes qu'il apportait tous les dimanches au second, qui les classait dans trois paniers : «à tourner », « refusées », « hésitations ». On retrouve des éléments de Je t'aime... dans les deux dictionnaires (voir par exemple les entrées " Chat » et « Correspondance » dans le Dictionnaire des idées revues) ; mais on peut faire l'hypothèse que ces ouvrages puisent aussi et surtout dans les deux autres paniers.

Le Dictionnaire du mépris compte 443 entrées (d' « Absurde » à «Zoo » en passant par « Minable ») ; celui des idées revues propose environ 2000 noms communs (d' «Abattoir » à «Zut » en passant par « Morbide»), auxquels s'ajoutent une section «pages roses» (en fait pages grises, comportant 550 entrées elles aussi rangées alphabétiquement) et environ 500 noms propres qui, tel le fameux

1 Court extrait de film, sans date ni auteur, mais peut-être d'abord diffusé par la télévision belge en mars 1962 : URL : https://www.dailymotion.com/video/x1wh2t ; consulté pour la dernière fois le 8.02.2019. Sur le Solex voir DDM : « Camelote » et DIR : «Combien ».

2 Dictionnaire du mépris, Paris, Calmann-Lévy, 1973 ; Dictionnaire des idées revues, illustré par R. Topor, Paris, Denoël, 1985. Ci-après DDM et DIR, respectivement. 
homonyme Joseph von Sternberg ( « véritable génie du cinéma », faux noble et vrai juif), sont objets d'affection, voire de respect - c'est-à-dire tout le contraire du mépris. Il s'agit d'un « Larousse intime », le Petit Larousse étant à la fois un modèle formel et un contre-modèle honni ${ }^{3}$.

\section{Mépris, révolte et vitriol}

Dans la foulée du Dictionnaire du mépris, Sternberg fut le directeur, rédacteur et secrétaire d'une revue intitulée Mépris ${ }^{4}$. Ainsi fichée comme un étendard au cœur de son œuvre, la notion reste cependant assez insaisissable. D'abord parce que les occurrences du mot sont rares.

C'est que le mépris n'est pas une fin en soi ; il est l'étape d'une révélation; il ne vaut qu'en tant que constitutif d'un mode d'être au monde plus général : l'humour. Car « le véritable humour » est « fait de mépris, de révolte et de vitriol » (DDM, « Poison »). Ce mépris qui conduit sur la voie d'une juste appréciation des choses a un synonyme : dégoût. «Avant d'en arriver au goût, il faut passer par le dégoût » ( $D D M$, « Goût ») ; de même qu'il faut passer par le mépris pour arriver à l'humour.

Dans le contexte pourtant favorable de la France de De Gaulle et de Pompidou, le mépris sternbergien s'oppose à l'action politique ; s'il est insurrection, celle-ci est, sinon tout à fait intérieure, du moins strictement verbale et individuelle. D'ailleurs aucune allusion, dans les Dictionnaires ou dans la revue Mépris, à Mai 68, aux conflits sociaux, à l'action collective. Pas d'indignation, parce que l'indignation suppose l'espoir d'un changement. Car l'humour « ne fait jamais que dynamiter, détruire, mais ne propose aucune solution rien en échange » (DIR, « Humour »).

Outre l'entrée " Humour », le Dictionnaire du mépris contient trois autres occurrences du mot mépris. L'entrée « Mépris » (reprise dans le DIR) parle de celui que manifestent les flics et les putes en tutoyant les gens. L'entrée « Virilité » (elle aussi reprise en 1985) se réjouit que le MLF ait remis au goût du jour « la haine

\footnotetext{
3 Voir DDM, entrées « Image », « Lacune », « Putride ». Mon exemplaire d'occasion du DIR a été « retiré des collections de la bibliothèque de la ville de Fontenay-sous-bois », après avoir été emprunté 6 fois entre février 1986 et février 1987 (dont 5 fois par le même lecteur). Sans doute la misogynie et quelques autres provocations de Sternberg ne sont-elles plus de mise dans cette bibliothèque devenue Médiathèque Louis Aragon, dont, à en juger par son site internet, la lecture ne semble pas le souci premier.

4 MEPRIS, la revue qui n'a strictement rien à vendre, ou à louer. 3 numéros bimestriels de 80 pages, octobre et décembre 1973, février 1974, Kesserling éditeur, Yverdon, Suisse. Méprix : 9 francs. Principaux contributeurs : Gabriel Bacri, Berner, E. M. Cioran, Théophraste Épistolier, André Frédérique, Jean Gourmelin, Gérard Klein, Ring Lardner, Martial Leiter, Jacques Loew, Luc NissetRaidon dit Lucques, Claire Parenti, Patrick Roequiers, Roland Topor (auteur de la couverture du no 1 reprise en 1981 pour une célèbre affiche d'Amnesty International), Pierre Veber. « Rolf Kesserling attiré par le fantastique, l'anarchie et le délirant, fit faillite en France et retourna en Suisse » (DIR, s.v. « Leiter »).
} 
de l'homme, l'injure et le mépris » mais estime que la femme libérée vise mal et trop mollement : " le meilleur ennemi du mâle, c'est encore l'homme ». L'entrée « Silence » enfin, admire « la force de percussion du silence, du mépris, de l'indifférence » de Dieu, dont les hommes politiques, toujours trop bavards, seraient bien avisés de s'inspirer. Or, on remarque qu'aucune de ces formes de mépris ne correspond vraiment au « véritable humour » défini plus haut.

Un article anonyme de l'Encyclopédie de Diderot et D'Alembert distinguait trois sortes de mépris, selon que celui-ci est dirigé vers un supérieur, un égal ou un inférieur - l'insolence, la fierté et la hauteur - qui toutes trois procèdent de «l'estime de soi $\Downarrow^{5}$. Mais le mépris sternbergien ne manifeste aucune estime de soi, car le contempteur s'inclut dans les cibles contemptibles. Son humour méprisant, révolté et vitriolique trouve sa source non dans la jouissance d'une quelconque supériorité, ni dans une forme de gaieté à la Alphonse Allais, suscitée par l'absurdité du réel, mais bien dans la tristesse. Noir de préférence, gris à la rigueur, l'humour est bien « la politesse du désespoir », expression que Sternberg attribue à Chris Marker ${ }^{6}$.

C'est ainsi que Sternberg refuse l'ironie (qui «n'a rien à voir avec l'humour» et qui " est même son contraire) et espère atteindre le " véritable humour ", que, paraphrasant Lichtenberg, il décrit comme « un couteau qui n'aurait pas de manche mais deux lames dont la plus dangereuse se retournerait de préférence vers le ventre de son propriétaire $»^{7}$.

\section{Un anti-panthéon}

Entre 1973 et 1985, la France a changé et le Dictionnaire des idées revues porte la trace de ces changements déplorables (informatique, carte bleue, crainte nouvelle de la pollution, soutien de la gauche à l'insurrection polonaise...), mais les cibles restent les mêmes : l'automobile, la banque, le cinéma français, l'armée, la police, la religion, le sport, les surréalistes, la télévision, le théâtre, l’université.

Le mépris vise des auteurs « injustement connus »-tels Michel Audiard, Hervé Bazin, Maurice Druon, Françoise Sagan, Henri Troyat, François Truffaut auxquels s'oppose un panthéon moderne composé pour l'essentiel de dessinateurs humoristes, de jazzmen, de romanciers américains. Outre divers illustrateurs, les rares Français à trouver grâce aux yeux de Sternberg sont Céline, Darien, Ionesco,

5 D. Diderot, Encyclopédie, Paris, Samuel Fauche, 1751, p. 357.

6 Dans sa préface à une édition française du Dictionnaire du diable d'Ambrose Bierce (Nouvelles éditions Oswald, 1987). La paternité est fort disputée : Tristan Bernard, André Breton, Achille Chavée, Émile Cioran, Pierre Dac, Jean Giraudoux, Georges Ionesco, Georges Duhamel, Kierkegaard, Paul Valéry, Boris Vian. Les tenants de Chris Marker renvoient à un numéro anthologique spécial de la revue La Nef, consacré, en décembre 1950-janvier 1951, à l'Humour poétique.

7 «Ironie », DIR, p. 113 et Georg Christoph Lichtenberg, « Inventaire d'une collection d'objets » [1798], in Le Couteau sans lame et autres textes satiriques, trad. fr. Ch. Le Blanc, José Corti, 1999, p. 54. 
Lautréamont et Proust, ainsi que la plupart des figures du nouveau roman (Alain Robbe-Grillet, Claude Simon, Michel Butor).

Face aux figures méprisables représentatives de la prétention parisienne, Sternberg fait aussi un discret éloge de sa Belgique natale. Dans la dernière partie du Dictionnaire des idées revues, une place est accordée à Brel, à Brueghel (un Anversois, peintre de la vulgarité populaire et annonciateur des charniers nazis), à Delvaux, Magritte, Michaux, Félicien Rops (non seulement belge mais wallon), et Simenon. Sternberg s'identifie un peu à ce dernier, graphomane autodidacte né à Liège, mais se reconnaît surtout dans ses romans : «j'étais moi-même un personnage de Simenon, dans le courant des années 45 : un minable représentant en livres qui n'arrivait jamais à placer sa camelote $»$.

\section{Le mépris par alphabet}

Le mépris selon Sternberg se caractérise cependant moins par ses objets, généralement plutôt convenus, que par sa forme. La forme du dictionnaire, peut-être parce qu'elle prend acte non seulement du désordre du monde, mais du caractère arbitraire de tout ordre, a toujours eu partie liée avec le mépris. On peut citer l'Alphabet de l'imperfection et malice des femmes de Jacques Olivier (1617) ; le Grand Dictionnaire des pretieuses : historique, poetique, geographique, cosmographique, cronologique, armoirique [...] d'Antoine Baudeau de Somaize (1661) ; le Dictionnaire néologique à l'usage des beaux esprits de Desfontaines (1726) ; ou, plus près de nous et l'année même de la publication du Dictionnaire des idées revues, le Dictionnaire superflu à l'usage de l'élite et des bien nantis de Pierre Desproges (Seuil, 1985). Sternberg s'inscrit généralement dans cette tradition, mais il a surtout trois modèles, plus ou moins avoués : Voltaire, Flaubert et Bierce.

En 1764, paraît chez Gabriel Grasset, à Genève, la première édition du Dictionnaire philosophique portatif, rééditée en 1769 chez Gabriel Cramer, toujours à Genève, sous le titre La Raison par alphabet. Le livre de Voltaire compte alors 118 articles, d'Abbé à Vertu. Les Questions sur l'Encyclopédie, publiées l'année suivante en 9 volumes, comptent 410 articles, dont une cinquantaine tirés du Dictionnaire philosophique ${ }^{8}$. Les Questions sur l'Encyclopédie sont donc à la fois une sélection et une expansion considérable du Dictionnaire philosophique, tout comme le Dictionnaire des idées revues par rapport au Dictionnaire du mépris. Outre cette histoire éditoriale, Voltaire et Sternberg partagent une sorte de misanthropie radicale. Pourtant le second ne cite jamais le premier, pas même pour le critiquer. La raison profonde de cette censure tient sans doute à ce que nous disions plus haut sur la conception de l'humour. Quand Voltaire écrit à D'Alembert : « Portez-

8 Les articles non repris sont regroupés dans différentes éditions des O.C. de Voltaire sous le titre « Fragments sur divers sujets, par ordre alphabétique ». 
vous bien, éclairez et méprisez le genre humain » (19 février 1757), ou : « Je vous recommande beaucoup de courage et beaucoup de mépris pour le genre humain » (8 avril 1771), il est difficile de décider s'il s'inclut dans « le genre humain ». Mais d'autres textes précisent qu'il pense appartenir à « un petit troupeau séparé qu'on appelle la bonne compagnie ; ce petit troupeau étant riche, bien élevé, instruit, poli, est comme la fleur du genre humain $»^{9}$. Or, l'idée même d'une bonne compagnie est aux antipodes de la pensée de Sternberg, pour qui, dans un monde " composé de fripons, de fanatiques et d'imbéciles $»^{10}$, il n'y a pas de refuge.

Dans les deux livres que nous considérons ici, la présence de Flaubert est réduite à quatre citations des pages grises du Dictionnaire des idées revues, mais le titre même de ce livre est une référence évidente au Dictionnaire des idées reçues ou Catalogue des opinions chics. Le mépris tient une grande place dans cette œuvre posthume à laquelle Flaubert avait travaillé de 1850 à 1880 : «ÉPICURE. Le mépriser »; « ÉRUDITION. La mépriser comme étant la marque d'un esprit étroit ». Il convient aussi de mépriser : les bas-bleus, les chevaux de course, le dessert, les doctrinaires, les hobereaux de campagne, les mazarinades, les virtuoses... Et le dictionnaire de Flaubert est suivi d'une compilation de citations d'auteurs célèbres, dont : "Voltaire est infiniment méprisable (Louis Veuillot) ». Mais, à la différence de celui constitué par Sternberg, ce florilège est conçu comme un recueil d'inepties. En effet, Flaubert s'en prend aux méprisants, dans la mesure où mépriser, c'est participer à la bêtise bourgeoise. Le mépris n'est pas une arme flaubertienne; c'est une cible.

Contrairement à Voltaire et à Flaubert, Ambrose Bierce est une référence constante chez Sternberg. Son Dictionnaire du Diable (The Devil's Dictionary), recueil de 998 définitions, est le fruit d'un travail intermittent de vingt-cinq années, publié en 1911. L'écrivain américain a droit à une notice très élogieuse dans la troisième section du Dictionnaire des idées revues :

BIERCE (Ambrose) - Figure de proue de l'humour noir, Bierce est évidemment le plus grand oublié de l'Anthologie de l'humour noir de Breton qui, manifestement ne connaissait presque rien de la littérature d'Outre-Atlantique. — «Bitter Bierce » eut une vie tragique en harmonie avec la noirceur et la neurasthénie qui colorent tous ses écrits, principalement des nouvelles de guerre ou d'épouvante. [...] Son Dictionnaire du Diable demeure une œuvre inégalée où il distille à doses perverses un superbe mépris du genre humain, une froideur inébranlable et un cynisme impossible à entamer.

Et, en effet, certaines définitions de Bierce pourraient sortir de la plume de Sternberg («Homme (n.) : Animal dont l'occupation principale consiste à exterminer les autres animaux et ceux de son espèce... ») et il y a plus d'une

\footnotetext{
9 Voltaire, Conversation de M. l'intendant des menus en exercices avec M. l'abbé Grizel (1761), in Euvres complètes de Voltaire, éd. L. Moland, Paris, Garnier, t. 24, 1879, p. 247.

${ }^{10}$ Ibid.
} 
ressemblance entre le nouvelliste autodidacte prolifique à la « froideur inébranlable » et l'auteur des Contes glacés ${ }^{11}$. Sternberg ressent sans doute une forme de fraternité avec celui qui eut vingt ans pendant la guerre de Sécession, qui a fait l'expérience du carnage, et qui, comme lui, est avant tout un rescapé. Mais le mépris est une passion qui n'est guère plus valorisée par Bierce que par Flaubert : « Mépris, n. Le sentiment d'un homme prudent face à un ennemi trop formidable pour qu'on s'oppose à lui $»^{12}$.

Sternberg s'inscrit donc dans une tradition particulière de misanthropie littéraire. La forme du dictionnaire correspond à une vision du monde qui prétend exclure les hiérarchies et placer chacun à la place aléatoire que lui assigne l'alphabet. Mais il s'écarte de cette tradition: d'abord parce qu'il revendique explicitement le mépris, passion généralement jugée mauvaise par ceux-mêmes qui la pratiquent ; ensuite parce qu'à l'arbitraire de l'alphabet, il n'hésite pas à en ajouter un autre, celui du calembour. Ce qu'il nomme le « charme discret de la linguistique » consiste à trouver dans le signifiant d'un mot la justification de la méfiance qu'il doit inspirer. Par exemple :

MÉMOIRE. Ce pourrait bien être une astucieuse inversion pour " mère du moi »; MILITANT. Débilitant serait plus conforme à la réalité. Et puis le terme « militant» évoque trop fâcheusement militaire pour inspirer confiance. - MAÎTRESSE. Ce n'est pas sans raisons sournoises que le mot « maîtresse » m'a toujours paru répugnant. Étonnant, le nombre de termes sordides qu'il recèle dans ses quelques syllabes : maître, masse, mères, ratissé, tir, mire, ire, âme, émir, mire, trime, miss, mise, armes, reître, tamisé, remisé, sire, tiers, métier, rétamé, terme, marié, amer, rets, mitre, as, S.S., rite, taré, tiré, messe, tassé, trié, mérite, restes, et il en reste sans doute un certain nombre ${ }^{13}$.

Cet exercice, assez proche de ce que l'on nomma au dix-huitième siècle le logogryphe, a pour double effet d'aggraver la condamnation par une sorte de preuve cratylienne du jugement porté, mais aussi de jeter la dérision sur l'acte même de condamner.

\section{Généalogie du mépris}

Plus développé que le $D D M$, plus enclin au jeu de mots, le $D I R$ est aussi nettement plus autobiographique. Dès l'Avant-propos : « J'ai barbouillé des milliers de pages et jamais, nulle part, je n'ai fait la moindre allusion à mon père ». Ce père, diamantaire anversois d'origine polonaise, est retourné en Pologne pour y mourir, à Majdanek, un camp au sud de Lublin, où 253000 déportés, dont 118000 juifs,

\footnotetext{
11 J. Sternberg, Contes glacés, André Gérard Marabout, 1974.

12 « Contempt, $\mathrm{n}$. The feeling of a prudent man for an enemy who is too formidable safely to be opposed ».

${ }^{13}$ DIR, p. 134, 137, 130.
} 
furent exterminés. Un peu plus loin : «L'assassinat sadique de cet homme épris de justice, aura fait de moi un être dégoûté, méprisant, à jamais ennemi de la race humaine ». Après un silence de soixante années, Sternberg se résout ainsi à donner la clé biographique qui explique sa vie et son œuvre.

Cette histoire familiale pourrait être rapprochée de celle de deux autres artistes français d'origine polonaise, deux amis de Sternberg : Topor et Perec. Roland Topor (1938-1997), fils d'un peintre et sculpteur immigré juif polonais, se cacha en Savoie avec ses parents pendant l'Occupation. Georges Perec (1936-1982) fut lui aussi caché dans les Alpes, mais sans ses parents, eux aussi juifs polonais, son père ayant été tué sur le front en juin 1940 et sa mère à Auschwitz en 1943. Mais, à la différence de Topor et Perec, Sternberg a été livré à lui-même au cœur de la guerre et du nazisme. C'est pourquoi son second dictionnaire peut être lu comme une enquête sur les racines intimes de son mépris. Après avoir évoqué les purs aryens qui envahirent la France en 1940, après avoir moqué les « incurables béats » qui s'imaginent que l'orchestre de déportés qui les accueille sur le quai d'un camp d'extermination « prouve qu'on organisera de petites fêtes dans ce lieu public », Sternberg livre un portrait de l'humoriste en jeune couard :

Durant la guerre, je n'ai échappé à travers tout qu'en respectant avec une constante vigilance une règle de base : ne penser qu'à la survie dans la lâcheté, ne jamais céder à la tentation de l'héroïsme. Toujours paniqué, plus prudent que nature, apolitisé, complètement égocentrisé sur ma rage de survivre, prêt à fuir, j'ai ainsi sauvé ma peau. Et quand, alors que la fin de la guerre s'amorçait, j'ai failli me faire tuer bêtement dans le maquis par une des dernières balles perdues, j'ai immédiatement déserté ${ }^{14}$.

Le mépris de soi est indissociable du mépris du monde, et Sternberg porte ce crachat au revers de son veston comme d'autres la légion d'honneur : «Il y a de ces mots qui attirent le putride. Légion par exemple : légion romaine, Légion étrangère, légion des Waffen S.S., Légion d'honneur. Autant de lésions d'horreur ${ }^{15}$.

\section{Bibliographie}

Bierce, Ambrose, The Devil's Dictionary, New York et Washington, Neale Publishing, 1911

Desfontaines, Pierre François Guyot, Dictionnaire néologique à l'usage des beaux esprits du siècle, s. $1 ., 1726$

Desproges, Pierre, Dictionnaire superflu à l'usage de l'élite et des bien nantis, Paris, Seuil, 1985

Diderot, Denis, Encyclopédie, Paris, Samuel Fauche, 1751

Flaubert, Gustave, Dictionnaire des idées reçues. Catalogue des opinions chics, posthume, Paris, Louis Conard, 1913

Humour poétique, numéro spécial de la revue La Nef, Paris, Librairie Jules Tallandier, décembre 1950-janvier 1951

${ }^{14}$ DIR, entrées « Blond », « Béatitude » et « Couardise ».

${ }^{15}$ DIR : « Légion ». 
Olivier, Jacques, Alphabet de l'imperfection et malice des femmes, Paris, 1617

Somaize, Antoine Baudeau de, Le Grand Dictionnaire des prétieuses, historique, poétique, géographique, cosmographique, chronologique et armoirique, Paris, Ribou, 1661

Sternberg, Jacques, Dictionnaire du mépris, Paris, Calmann-Lévy, 1973

Sternberg, Jacques, Contes glacés, Verviers, André Gérard Marabout, 1974

Sternberg, Jacques (éd.), MEPRIS, la revue qui n'a strictement rien à vendre, ou à louer, 3 numéros, octobre et décembre 1973, février 1974, Yverdon, Kesserling éditeur

Sternberg, Jacques, Dictionnaire des idées revues, illustré par Roland Topor, Paris, Denoël, 1985

Sternberg, Jacques, préface au Dictionnaire du diable d'Ambrose Bierce, Nouvelles éditions Oswald, 1987

Voltaire, Conversation de M. l'intendant des menus en exercices avec M. l'abbé Grizel (1761), in Euvres complètes de Voltaire, éd. Louis Moland, Paris

Voltaire, Correspondance, in $O C$, éd. Moland, Garnier, t. 33-50, 1880-1882

Voltaire, Dictionnaire philosophique portatif, Genève, Gabriel Grasset, 1764

Voltaire, La Raison par alphabet, Genève, Gabriel Cramer, 1769

Voltaire, Questions sur l'Encyclopédie, par des amateurs, 9 vol., Genève, Cramer, 1770-1771

Denis Reynaud est professeur émérite de littérature française du XVIII ${ }^{\mathrm{e}}$ siècle. Il a enseigné une dizaine d'années en Angleterre, aux États-Unis et au Japon, puis à l'université Lyon 2 où il fut doyen de la Faculté des Lettres. Son activité de recherche porte principalement sur la presse périodique avant et pendant la Révolution. Il est responsable du site http://www.gazettes18e.fr/. Il a publié, avec Delphine Gleizes, Machines à voir. Pour une histoire du regard instrumenté, 2017 ; et, avec Martine Boyer-Weinmann, Le Vestiaire de la littérature, 2019.

\begin{tabular}{|c|c|}
\hline & $\begin{array}{l}\text { (C) by the author, licensee Łódź University - Łódź University Press, } \\
\text { Łódź, Poland. This article is an open access article distributed under } \\
\text { the terms and conditions of the Creative Commons Attribution license } \\
\text { CC-BY-NC-ND } 4.0 \text { (https://creativecommons.org/licenses/by-nc-nd/4.0/) }\end{array}$ \\
\hline & Received: 2019-01-20; Accepted: 2020-12-18 \\
\hline
\end{tabular}

